\title{
Measuring Beliefs in Centimeters: Private Knowledge Biases Preschoolers' and Adults' Representation of Others' Beliefs
}

\author{
Jessica A. Sommerville \\ University of Washington
}

\author{
Daniel M. Bernstein \\ Kwantlen Polytechnic University and \\ University of Washington
}

\author{
Andrew N. Meltzoff \\ University of Washington
}

\begin{abstract}
A novel task, using a continuous spatial layout, was created to investigate the degree to which (in centimeters) 3-year-old children's $(N=63)$, 5-year-old children's $(N=60)$, and adults' $(N=60)$ own privileged knowledge of the location of an object biased their representation of a protagonist's false belief about the object's location. At all ages, participants' knowledge of the object's actual location biased their search estimates, independent of the attentional or memory demands of the task. Children's degree of bias correlated with their performance on a classic change-of-location false belief task, controlling for age. This task is a novel tool for providing a quantitative measurement of the degree to which self-knowledge can bias estimates of others' beliefs.
\end{abstract}

Successful perspective taking in the everyday world hinges on the ability to understand that others have beliefs, desires, and emotions that can differ from, and even contradict, one's own mental states. Research conducted under the umbrella of "theory of mind" has made impressive gains in uncovering the developmental origins of the understanding that others can have beliefs that differ from reality, as measured in the classic false belief task. Based on this work, many researchers have argued that there is a qualitative shift between 3 and 5 years of age in children's understanding that beliefs are representations of the world, not reflections of reality, and therefore can be incorrect and/or incomplete

This work was generously supported by grants from the National Science Foundation (SBE-0354453) and the Social Sciences and Humanities Research Council of Canada (SSHRC 4102008-1681). Any opinions, findings, and conclusions expressed in the study are those of the authors and do not necessarily reflect the views of NSF or SSHRC. We are grateful to the parents and children who participated in this research. We would like to thank Joy Durham, Jill Huynh, Reema Jayaker, Leah Lee, Jacque Mullen, Atsuko Ishida, and Sarah Zebrowski for their assistance with the study, and Betty Repacholi, Yuichi Shoda, and Cheryl Kaiser for their comments on an earlier version of this manuscript.

Correspondence concerning this article should be addressed to Jessica A. Sommerville, Department of Psychology, Box 351525, University of Washington, Seattle, WA, 98195, or Daniel M. Bernstein, Department of Psychology, Kwantlen Polytechnic University, 12666-72nd Ave., Surrey, BC, V3W 2M8, Canada. Electronic mail may be sent to sommej@u.washington.edu or Daniel.Bernstein@ kwantlen.ca. (e.g., Gopnik \& Astington, 1988; Perner, 1991; Wellman, 1990; Wimmer \& Perner, 1983).

Adults and older children pass classic change-oflocation false belief tasks, demonstrating that they appreciate that others can hold false beliefs. Nevertheless, it is the case that even in adults, one's own knowledge can contaminate or distort one's understanding of another person's beliefs. Indeed, many studies have demonstrated that adults' own knowledge and beliefs significantly bias their understanding of the beliefs and knowledge of other people (e.g., Apperly, Back, Samson, \& Grant, 2007; Apperly, Warren, Andrews, Grant, \& Todd, 2011; Back \& Apperly, 2010; Bernstein, Erdfelder, Meltzoff, Peria, \& Loftus, 2011; Birch \& Bloom, 2004, 2007; Chambers, Epley, Savitsky, \& Windschitl, 2008; Epley, Keysar, Van Boven, \& Gilovich, 2004; Mitchell, Robinson, Isaacs, \& Nye, 1996; Nickerson, 1999; Royzman, Cassidy, \& Baron, 2003).

Our goal was to create a new task that measures the degree to which children's and adults' own knowledge of an object's actual location biases their estimates of where a protagonist, who holds a false belief about the object's location, will search for the object. We devised a novel task that capitalizes on

(C) 2013 The Authors

Child Development (c) 2013 Society for Research in Child Development, Inc. All rights reserved. 0009-3920/2013/xxxx-xxxx

DOI: $10.1111 /$ cdev. 12110 
aspects of the classic change-of-location task but departs from the classic task in important, theoretically motivated ways. As in the classic change-oflocation task, child and adult participants are told a story in which a protagonist places an object in a hiding location before leaving the room, and while she is gone her companion moves the object to a new hiding location. However, unlike the classic change-of-location task, which uses two well-marked discrete hiding locations (e.g., a box and a cupboard), in our novel task the protagonist buries the object in an unmarked location in a continuous space (a sandbox), and her companion moves the object to a second unmarked location in the same continuous space while the protagonist is absent. Then, following a short delay, the child or adult participant must indicate where, within the sandbox, the protagonist will look for the object once she returns.

Whereas the classic change-of-location task is designed to assess whether participants appreciate that a protagonist can hold a false belief, our Sandbox task focuses on a different but related issue. The goal of our task is to test the degree to which participants' knowledge of the object in its new location biases their representation of where the protagonist thinks the object is located. Thus, our task is designed to focus on the amount of bias (measured in centimeters) that the participants' own privileged knowledge exerts on their representation of another person's belief about a location in space. If the participants' own knowledge biases this representation, they should predict that the protagonist will not believe that the object is where they last saw it, but at a location in the sandbox that is biased closer to the object's actual location (i.e., where the participant knows the object to be). Through the use of a continuous spatial layout, our task enables us to measure the amount of participants' bias in centimeters. Past work has used a sandbox to investigate systematic biases in how children represent space (Huttenlocher, Newcombe, \& Sandberg, 1994). Using the sandbox in the context of a perspective-taking task may provide a means to uncovering interesting issues in adults' and children's representations of other people's beliefs.

Given past work demonstrating that even adults have difficulty suppressing or ignoring their own knowledge to reason from a more ignorant perspective (e.g., Apperly et al., 2007; Bernstein et al., 2011; Birch \& Bloom, 2004, 2007; Epley, Morewedge, \& Keysar, 2004; Keysar, Lin, \& Barr, 2003), we expect that both children and adults will show systematic biases in their search estimates indicating that their estimates are contaminated by their knowledge of the object's actual location. Although the classic change-of-location task cannot be used in its traditional form with adults (because of ceiling effects), our novel Sandbox task can be used across a large range of ages. Thus, our task provides an innovative way to measure developmental changes, and individual differences, in representational biases using a single task.

Our new task is also important because of its ecological validity; in the real world, perspective taking generally, and false belief situations more specifically, do not always involve two well-marked hiding locations and immediate estimates of others' beliefs. In the classic change-of-location false belief task, the hiding locations are well known (e.g., a box and a cupboard) and well marked, and there is minimal (if any) delay between the vignette and participants' judgments of where the protagonist will search for the hidden object. However, in the real world there are often instances when one's memory of the original location of an object or the new location of an object is less precise. Moreover, estimates about others' beliefs are not always instantaneous. Nonetheless, it is useful, and often necessary, to be able to estimate what another person believes in these cases. Thus, our task may provide insights into how adults and children estimate or predict what others know "in the wild" (see also Moll \& Meltzoff, 2011).

In two experiments, we used our novel Sandbox task to investigate 3-year-olds', 5-year-olds', and adults' ability to estimate where a participant would search for a hidden object in a continuous spatial layout. Participants in Experiment 1 completed one of two conditions. In the false belief condition, participants had to predict where another person would look for an object based on that person's false belief. Participants also completed a control condition with similar task demands that did not involve reasoning from the protagonist's (differing) perspective. For participants in the false belief condition, we predicted that all age groups would be biased by their own knowledge. In addition, we predicted a monotonic reduction in the extent of this bias with age, based on prior work (Bernstein et al., 2011; Birch \& Bloom, 2004, 2007). Child participants also completed a classic false belief task involving a change in location using discrete, well-marked locations, so we could examine relations between our Sandbox task and the standard false belief task. 


\section{Experiment 1}

\section{Method}

Participants. Forty 3-year-olds $(20$ girls, $M=$ 41 months, $S D=2.5$, range $=37-47$ months), forty 5-year-olds $(20$ girls, $M=67$ months, $S D=3.0$, range $=61-71$ months), and 40 college students (20 females) participated in the experiment. Participants were from a large city in the Pacific Northwest. Child participants were recruited from a university-maintained database; adult participants received course credit. Information on ethnicity and socioeconomic status was not formally recorded, although the majority of participants were Caucasian. All participants were fluent English speakers.

Participants were randomly assigned to the false belief condition or the no false belief condition ( $n=20$ per age group and condition). Eight additional participants were tested and excluded from the study because they did not complete the testing procedure ( $n=4$ children) or because they failed to follow instructions ( $n=4$ adults).

Procedure. We based our Sandbox task on a hiding task developed by Huttenlocher et al. (1994), which used a 5-ft-long homogenous sandbox to investigate young children's ability to code spatial location. Participants were tested using a rectangular sandbox constructed from pine $(152 \mathrm{~cm}$ long $\times$ $45 \mathrm{~cm}$ wide $\times 31 \mathrm{~cm}$ deep). The sandbox was filled with Styrofoam peanuts to $2.5 \mathrm{~cm}$ below the top lip. The sandbox was painted gray with a green base and sat upon a table. Children were seated on an adjustable height swivel chair in front of the sandbox. The chair was aligned with the midpoint of the sandbox, and the top lip of the sandbox was approximately waist high to an adult. Adults stood in front center of the sandbox. The experimenter stood directly across from participants on the other side of the sandbox.

The experimenter, unaware of the experimental hypotheses, narrated a story and placed objects within the sandbox while participants watched. In these stories a protagonist put an object in one location, and while the protagonist was absent, a second character subsequently moved that object to a new location (false belief condition) or placed another object in the new location (no false belief condition). Participants were then asked, after a short delay, to indicate where the protagonist would search for the object upon her return. To illustrate, in one story participants were told, "Judy and her dad are planting flowers in the planter box to surprise her mom. Judy's dad buries the flower here (experimenter hides a plastic flower at the first hiding location) and then goes to the shed to find a shovel." The procedure then varied according to condition. Participants in the false belief condition were told: "While Judy's dad is gone, Judy decides to move the flower here," and the experimenter moved the flower to the second hiding location. Participants in the no false belief condition were told: "While Judy's dad is gone, Judy puts the plant food here," and the experimenter hid the plant food at the second hiding location. Participants in both conditions were then asked, "When Judy's dad comes back, where is he going to look for the flower?"

Each trial involved a different story. Thus, the box represented a different continuous hiding location on each trial (garden plot, sandbox, deep chest freezer, and bathtub) and featured a different set of characters and different hiding objects (hiding objects were miniature replicas of the objects mentioned in the story). Stories occurred in a fixed order (see http:/ / sandboxtask.weebly.com/ for the stories).

There were four trials consisting of two different trial types. On the two large distance trial types, $36 \mathrm{~cm}$ separated the first and second hiding locations; on the two small distance trial types, $15 \mathrm{~cm}$ separated the first and second hiding locations. One trial of each type involved a second hiding location either to the left or right of the first hiding location. Small and large distance trials alternated, with the first trial type counterbalanced across participants (see the Appendix for exact hiding locations). Each trial contained a different set of hiding locations. After each story, participants completed a 45-s distractor task, which we administered to prevent participants from using perceptual strategies to guide their search (e.g., by continuing to fixate on the hiding location). During the distractor task participants turned away from the sandbox and tried to find Waldo in a Where's Waldo? book. The experimenter also leveled the Styrofoam peanuts during this time to ensure that participants could not use surface perturbations to guide their search estimates. After $45 \mathrm{~s}$, participants faced the sandbox and indicated where the protagonist would look for the object when he or she returned by pointing to the exact location on the surface of the Styrofoam peanuts. At the end of the testing procedure, the experimenter used a ruler to score participants' search responses on the task, using a set of concealed stickers on the experimenter's side of the box that indicated the participants' responses on each trial. Bias scores were calculated by determining the absolute value of the difference between the participants' search responses and the first hiding location and then assigning a positive or negative sign 
based on the direction of the second location. Thus, responses biased toward the second location were assigned positive values, and responses biased away from the second location were assigned negative values.

Children then took a short break before completing a standard change-of-location task as part of a theory-of-mind battery (see Bernstein, Atance, Meltzoff, \& Loftus, 2007). Subsequent analyses focused exclusively on the change-of-location task; correct answers on the standard change-of-location task received a score of 1 and incorrect answers received a score of 0 . Children who failed the control question ("Where is the object really?"; two 3-year-olds and one 5-year-old) were excluded from subsequent analyses involving the change-of-location task.

\section{Results and Discussion}

We excluded data from five 3-year-olds, four 5 -year-olds, and one adult because these subjects scored more than $2 S D$ above or below the mean on the small difference and/or large distance trials. Including these subjects did not change the overall data pattern.

A preliminary analysis revealed an effect of trial type, $F_{\mathrm{s}}(1,113)=23.7, p=.0001$; thus, we subsequently conducted separate univariate analysis of variance (ANOVA) on bias scores for small and large distance trials.

An ANOVA on large distance trials with belief (false belief vs. no false belief) and age (3-year-olds vs. 5 -year-olds vs. adults) as the between-subjects variables revealed main effects of belief, $F_{\mathrm{s}}(1,108)=39.7$, $p=.0001, \quad \eta_{\mathrm{p}}{ }^{2}=.27$, and age, $F_{\mathrm{s}}(2,108)=11.2$, $p=.0001, \eta_{\mathrm{p}}^{2}=.17$, and a Belief $\times$ Age interaction, $F \mathrm{~s}(2,108)=5.1, p=.01, \eta_{\mathrm{p}}{ }^{2}=.09$. An ANOVA on small distance trials with belief and age as betweensubjects variables also revealed main effects of belief, $F_{\mathrm{s}}(1,108)=28.5, p=.0001, \eta_{\mathrm{p}}^{2}=.21$, and age, $F_{\mathrm{s}}(2$, $108)=8.5, p=.0001, \eta_{\mathrm{p}}^{2}=.14$, and a Belief $\times$ Age interaction, $F_{\mathrm{s}}(2,108)=10.7, p=.0001, \eta_{\mathrm{p}}{ }^{2}=.17$. As Figures 1 and 2 indicate, the difference between bias scores in the no false belief and false belief conditions diminished with age. Bias scores were greater for the false belief condition than the no false belief condition on the large distance trials for 3-year-olds, $t(35)=4.3$, $p=.0001, d=1.44 ; 5$-year-olds, $t(36)=3.9, p=.0004$, $d=1.30$; and adults, $t(37)=2.3, p=.025, d=.76$, and on the small distance trials for 3 -year-olds, $t(35)=4.6$, $p=.0001, d=1.56$, and 5-year-olds, $t(36)=3.7$, $p=.001, d=1.23$; $n$ s for adults. In the false belief condition alone, bias scores on large distance trials differed between 3- and 5-year-olds, $t(34)=2.2, \quad p=.03$,

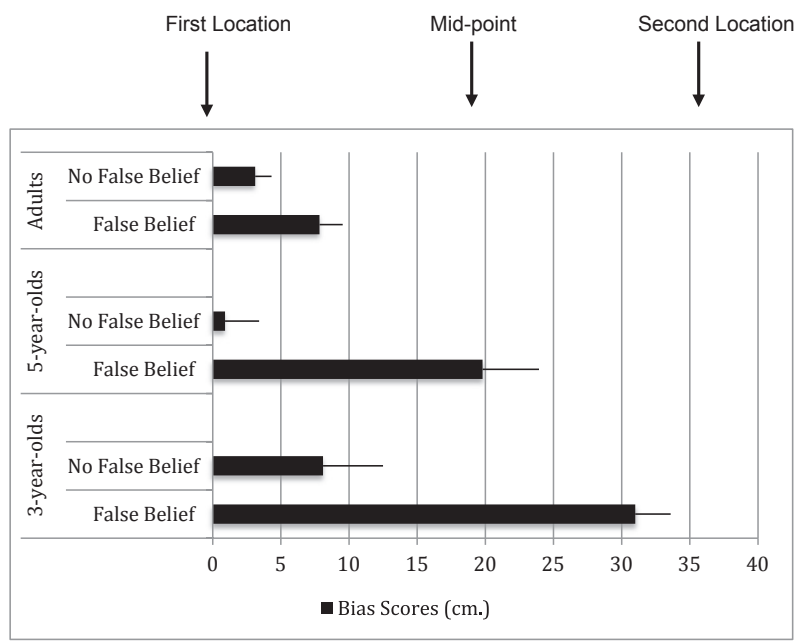

Figure 1. Mean large distance bias scores (standard error) as a function of age group and condition, Experiment 1.

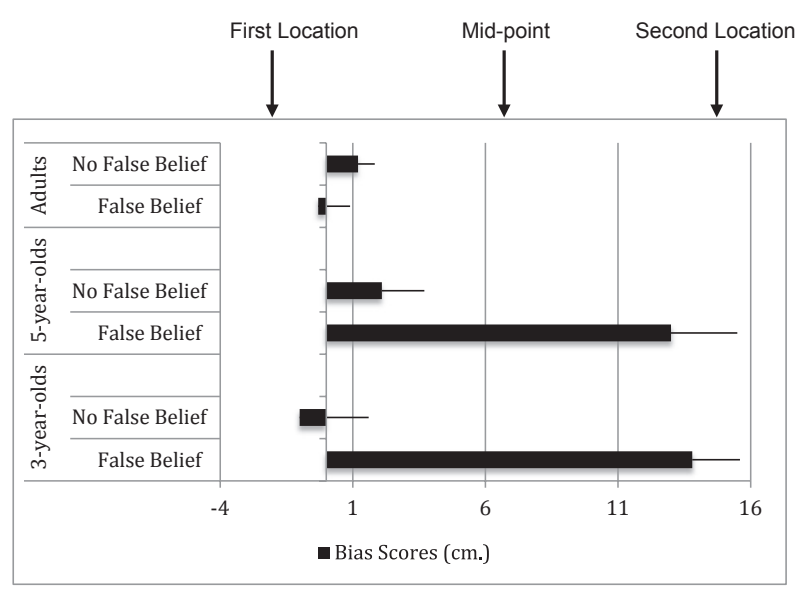

Figure 2. Mean small distance bias scores (standard error) as a function of age group and condition, Experiment 1.

$d=.76$; between 3-year-olds and adults, $t(34)=7.7$, $p=.0001, d=2.64 ;$ and between 5-year-olds and adults, $t(36)=2.7, p=.01, d=.90$. On small difference trials, bias scores in the false belief condition differed between 3-year-olds and adults, $t(34)=6.8, p=.0001$, $d=2.33$, and between 5-year-olds and adults, $t(36)=4.8, p=.0001, d=1.60 ; 3$ - and 5-year-olds did not differ from one another, $n s$. There were no significant differences between age groups for the no false belief condition, all $n s$.

To ensure that the difference between the false and no false belief conditions did not occur because the no false belief condition featured two separate objects, whereas the false belief condition featured a single object, we tested a separate group of 20 adults on another control condition in which the protagonist watched as the object was moved from 
the first to the second location. Again, participants indicated where the protagonist would search for the object. Bias scores were calculated by determining the absolute value of the difference between the second hiding location (the actual object location) and the first hiding location (responses biased toward the first location were assigned positive values). Planned comparisons revealed that bias scores for the false belief condition were significantly greater than in this new control condition for the large distance trials, $t(38)=2.2, p=.03, d=.71$. Bias scores on this control condition did not differ from the no false belief condition, $n s$.

Next, we assessed the relation between children's performance on the standard change-of-location task and the Sandbox task. Replicating prior work, 5 -year-olds outperformed 3-year-olds on the standard change-of-location task, $t(78)=5.2, p=.0001$, $d=.82$. Children's performance on the change-oflocation task was negatively related to their scores on the Sandbox task for children in the false belief condition, large distance trials: $r_{\mathrm{pb}}(35)=-.46$, $p=.006$; age partialed: $r_{\mathrm{pb}}(32)=-.39, p=.02$ : children who succeeded on the classic change-of-location task were less biased by the object's actual location in the Sandbox task. Performance on the change-of-location task was unrelated to performance on the no false belief condition in the Sandbox task, ns. These findings suggest that our novel task provides an assay of the extent to which children's (and adults') own knowledge of an object's actual location biases or contaminates their representations of another person's belief.

\section{Experiment 2}

The findings from Experiment 1 show that for both adults and children their own knowledge biases or contaminates their representations of the beliefs held by another person, and that this bias decreases with age. The results of the no false belief condition in Experiment 1 provide preliminary evidence that participants' difficulty involves perspective taking per se; the mere presence of a second hiding location did not bias participants' search estimates toward that location when the protagonist's belief did not pertain to the second object. This indicates that general attentional or cognitive demands of the task, such as having one's attention drawn to a second location or having to represent a second hiding location, do not account for enhanced bias scores in the false belief condition. However, the results of our task may have arisen from a more general diffi- culty considering an object's original location in the face of knowledge of its current location.

To rule out this possibility, we conducted a within-subjects experiment in which adult and child participants completed the false belief condition from Experiment 1 and a new memory condition in which participants recalled the object's initial location. Thus, although participants were required to provide the same piece of information across all trials, it was only on the false belief trials that they needed to adopt another person's perspective. To increase the generalizability of our findings to other false belief tasks, the delay was also reduced to $20 \mathrm{~s}$. As in Experiment 1 , children also received a classic change-oflocation false belief task following a short break.

\section{Method}

Participants. Adult participants were 20 college students (15 females) from a large city in the Pacific Northwest who participated in the experiment in exchange for course credit. Fourteen participants were Asian and six were Caucasian. Information on socioeconomic status was not recorded. All participants were fluent English speakers; 10 were native English speakers, and 10 were non-native English speakers. One additional participant was tested and excluded from the study because he or she failed to follow directions.

The child participants were twenty-three 3-yearolds (15 girls, $M=41$ months, $S D=4.2$, range = 36-47 months) and twenty 5-year-olds (16 girls, $M=62$ months, $S D=2.8$, range $=60-69$ months). Participants were recruited from a university-maintained database. Information on ethnicity and socioeconomic status was not formally recorded, although the majority of participants were Caucasian. All participants were fluent English speakers.

Procedure. Participants completed nine trials, each featuring a different story. Four trials were identical to those of the false belief condition of the Sandbox task of Experiment 1 (false belief trials): Participants predicted where the protagonist would look for the object upon his or her return. Four trials were memory trials in which participants indicated their memory of the object's original location after the protagonist returned (e.g., "Where did he put the ice cream before he left?"; see http://sandboxtask.weebly.com/expt2.html). Participants also completed a single switch trial, occurring in between the four false belief and four memory trials, in which the protagonist watched the object being moved to another location. We added this trial to keep participants from always choosing the first location. Given 
that Experiment 1 demonstrated enhanced bias scores on large-distance trials but not small-distance trials, all trials used a difference of $36 \mathrm{~cm}$ between the hiding locations. Half the trials involved movement of the object from left to right, and half from right to left (see the Appendix for exact hiding locations). Trials were blocked in groups of four; half the participants received the memory control trials first. After each story, participants completed a 20-s distracter task (identical to Experiment 1) and then provided their response by pointing to the exact location on the surface of the Styrofoam peanuts. The scoring method was identical to Experiment 1.

The procedure was identical for adult and child participants, except that children completed the classic change-of-location false belief task after they completed the Sandbox task.

\section{Results and Discussion}

Bias scores for the memory and false belief trials, calculated in the same manner as in Experiment 1, are presented in Figure 3 (performance on the switch trial was not analyzed). We excluded data for one adult because this subject scored more than $2 S D$ above the mean. Including this subject did not change the overall data pattern.

An ANOVA on bias scores with trial type as the within-subjects variable (false belief vs. memory trials) and age group as the between-subjects variable (3year-olds vs. 5-year-olds vs. adults) revealed a main effect of trial type, $F_{\mathrm{s}}(1,59)=11.62, p=.001, \eta_{\mathrm{p}}{ }^{2}=.17$, indicating that participants' bias scores were greater on false belief trials $(M=23.00, S E=1.84)$ than the memory trials $(M=16.91, S E=2.00), t(61)=3.47, p=.001$, $d=0.40$, and a main effect of age group, $F \mathrm{~s}(2$, $59)=45.24, p=.0001, \eta_{\mathrm{p}}{ }^{2}=.61$, indicating that overall bias scores varied as a function of age; the interaction between trial type and age group was not significant,

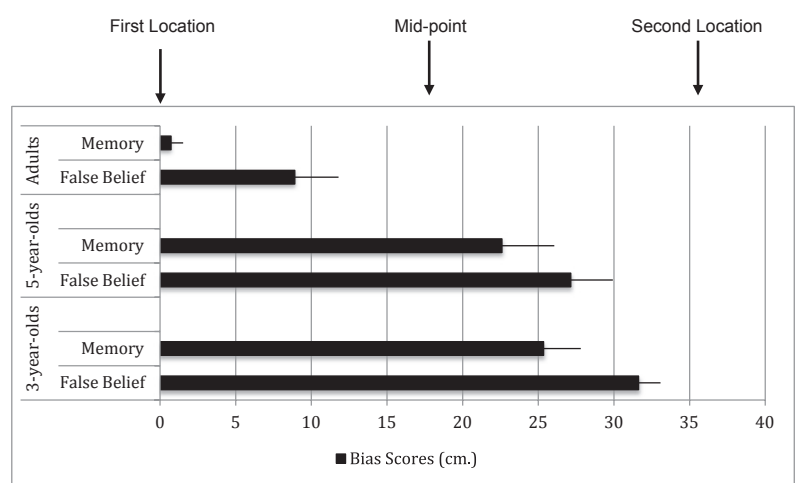

Figure 3. Mean bias scores (standard error) as a function of age group and condition, Experiment 2.
$F_{\mathrm{s}}(2,59)=.17, p=.85$. Adults $(M=4.22, S E=1.61)$, showed significantly less bias than 3-year-olds $(M=$ 28.50, $S E=1.61), \quad t(40)=10.58, p=.0001, d=3.30$, and 5-year-olds $(M=24.87, S E=2.45), t(37)=6.97$, $p=.0001, d=2.25$; overall bias scores did not differ between 3- and 5-year-olds, $t(41)=1.27, p=.21$.

We subsequently conducted planned comparisons across age groups for bias scores on the false belief trials and the memory trials. For the false belief trials, bias scores for adults $(M=8.91$, $S E=2.89)$ were significantly lower than for 3-yearolds $(M=31.63, S E=1.45), t(41)=7.31, p=.0001$, $d=2.19$, and 5-year-olds $(M=27.15 S E=2.8)$, $t(38)=4.55, p=.0001, d=1.44 ;$ bias scores did not differ between 3 - and 5-year-olds, $t(41)=1.48$, $p=.15$. For the memory trials, bias scores for adults $(M=0.73, S E=0.80)$ were significantly lower than for 3-year-olds $(M=25.36, S E=2.46), t(40)=8.76$, $p=.0001, d=2.82$, and 5-year-olds $(M=22.60$, $S E=3.5), \quad t(37)=6.02, \quad p=.0001, \quad d=1.95 ; \quad$ bias scores did not differ between 3- and 5-year-olds, $t(41)=.66, p=.55$. Planned comparisons within each age group comparing bias scores for false belief trials and memory trials revealed that both 3year-olds, $t(22)=2.56, p=.018, d=0.65$, and adults, $t(18)=2.78, p=.012, d=.79$, showed significantly more bias on false belief trials than memory control trials; for 5-year-olds although bias scores on the false belief trials were numerically higher on the false belief trials, this difference did not reach statistical significance, $t(19)=1.16, p=.26$ (presumably due to the fact that 5-year-olds' bias scores on the memory trials were bimodally distributed; see below).

A visual inspection of the data indicated that bias scores were unimodally distributed at each age group for the false belief trials, ensuring that a subset of the participants at each age was not accounting for overall bias scores (e.g., some participants showing strong bias, but others showing no bias). However, 5-yearolds' performance on the memory trials was bimodal: Whereas some 5-year-olds showed strong bias on memory trials, others showed less bias. In a subsequent analysis we subdivided 5-year-olds into two groups on the basis of their bias scores using the naturally observed split in the data: those with low memory bias scores $(M=5.13, S E=2.20$, range $=-1.43$ to $16.83)$ and those with high memory bias scores $(M=34.23, S E=1.23$, range $=26.67-39.69), t(18)=$ $12.47, p=.0001, d=5.47$, and compared these two groups on their false belief bias scores. This analysis revealed that false belief bias scores did not differ significantly for 5-year-olds with low memory bias $(M=25.46, \quad S E=5.52)$ versus those with high 
memory bias $(M=28.28, S E=3.02), \quad t(18)=.49$, $p=.63$. This finding suggests that poor memory of the first object location cannot account for individual differences in the degree of bias evident on false belief trials: Children who had a relatively good memory for the first location of the object showed just as much bias on false belief trials as children who had relatively poor memory for the first location of the object.

We next assessed the relation between children's bias scores on false belief trials and their performance on the classic change-of-location task. Replicating Experiment 1, children who succeeded on the classic change-of-location task were less biased by the object's actual location on false belief trials: $r_{\mathrm{pb}}(43)=-.38$, $p=.01$; age partialed: $r_{\mathrm{pb}}(40)=-.37, p=.02$. Critically, this relation was maintained when children's bias scores on the memory control trials were partialed out, $r_{\mathrm{pb}}(40)=-.34, p=.03$; age partialed. Taken together, these findings suggest that children's bias scores on the false belief trials are not merely driven by an inability to recall the original object location in the face of the object's current location.

\section{General Discussion}

Using a novel Sandbox task, we demonstrated that 3year-olds, 5-year-olds, and adults are biased by their own knowledge of an object's current location when asked to estimate where a protagonist would search for the object. At all ages, participants' assessment of the protagonist's belief was biased by the participants' own knowledge about the actual state of affairs in the world (e.g., toward the object's actual location). Moreover, in control conditions when adults and children were asked to estimate the original object location, rather than the protagonist's belief about the object's location, they showed significantly less bias (Experiment 2). These findings weigh against the possibility that having to suppress one hiding location in favor of another location solely accounts for increased bias scores in the false belief condition.

In Experiment 1, 3-year-olds tested in the false belief condition of the Sandbox task produced search estimates on both the small- and large-distance trials that closely approximated the actual location of the object, and were significantly greater than those of 5-yearolds. This is consistent with findings obtained on classic change-of-location tasks using discrete, well-marked locations and verbal responses (Wellman, Cross, \& Watson, 2001), and the claim that 3-year-olds do not yet understand that another person can hold a false belief. Three- and 5-year-olds' performance did not differ on the false belief trials in Experiment 2, although 5-year-olds' bias scores were numerically lower than those of 3-year-olds. This finding may have arisen because 5-year-olds in Experiment 2 were on average 5 months younger than 5-year-olds in Experiment 1 .

In both Experiments 1 and 2, 5-year-olds also exhibited difficulty on our Sandbox task. These findings diverge somewhat from those of standard change-of-location tasks in which 5-year-olds typically outperform 3-year-olds and achieve performance significantly above chance. These results have implications for children's perspective-taking abilities: Although 5-year-old children may understand the representational nature of beliefs, their own knowledge can still significantly contaminate their estimates of another person's beliefs, as shown in this task and others (e.g., Bernstein et al., 2007).

One important question concerns why 5-year-olds fare less well on our Sandbox task than they do on a classic change-of-location task. It is possible that the insertion of a delay in our novel Sandbox task produced this finding. However, we think that it is unlikely that this delay accounted for 5-year-olds' diminished performance with respect to the classic change-of-location false belief task. First, in Experiment 2, although children showed bias on the memory trials, this bias was significantly less than on the false belief trials. Second, a reduction in the response delay from $45 \mathrm{~s}$ (Experiment 1) to $20 \mathrm{~s}$ (Experiment 2) did not decrease bias scores on false belief trials (see Figures 1 and 3). Third, 5-year-olds with relatively good memory for the first location of the object did not differ from 5-year-olds with relatively poor memory for the first location of the object in their degree of bias on the false belief trials. Fourth, the relation between children's bias scores on false belief trials of the Sandbox task and the classic change-of-location false belief task was significant even when we controlled for bias on memory trials.

Rather, we think it is more likely that other aspects of our task led to 5-year-olds' diminished performance in comparison to the classic change-oflocation task, and that these factors are theoretically important. Whereas the use of discrete, wellmarked, well-known locations in the classic changeof-location task may assist children's ability to reason according to the protagonist's false belief by ensuring less representational blending of one location with another location, the use of a single continuous space in our task may make it harder for children to segregate their own knowledge of the object's actual location from that of the protagonist's belief about the object's location. Indeed, the findings from Experiment 1 suggest that 5-year-olds 
may find it particularly difficult to avoid contamination by their own beliefs when these are only subtly different from another person's beliefs (embodied in this experiment as a small distance between unmarked locations) versus when the content of beliefs is more distinct from that of another person (embodied in this experiment as a large distance between unmarked locations): On largedistance trials, 5-year-olds' search estimates were roughly halfway between the two hiding locations; on small-distance trials, 5-year-olds' search estimates approximated the second hiding location.

It is also possible that 5-year-olds' performance on our task was influenced by the type of labels used in the vignettes to refer to the hiding locations. Other authors have argued that language generally, and labels specifically, contribute to children's success on standard false belief tasks (see San Juan \& Astington, 2012, for a review). In our task because the two hiding locations did not correspond to discrete, nameable locations, similar nondistinct labels were used to refer to the locations (e.g., "Judy's dad buries the flower here ... Judy moves the flower here"), providing minimal linguistic support for solving the task.

Recently, Low and Simpson (2012) demonstrated that whereas $80 \%$ of 4 -year-old children pass the first trial of an explicit false belief task when the two discrete hiding locations are referred to with distinct labels (e.g., "the flower-pot" and "the bag"), only $33 \%$ of 4 -year-olds pass the first trial when the two discrete hiding locations are referred to with nondistinct labels (e.g., "here" and "here"; as in this study). Critically, whereas the use of distinct (vs. nondistinct) labels benefited 4-year-olds' performance in their study, the type of label used had no effect on 3-year-olds' performance. Hence, the use of nondistinct labels in this study, either independently, or in conjunction with the use of a continuous hiding space, may have affected older children's performance. As is the case when physical distances between hiding spaces are small, using nondistinct labels may lead to more representational bleed-over between the child's own beliefs and their representation of another person's beliefs. Alternately, given the hypothesized role of labels in children's cognitive flexibility (e.g., Jacques \& Zelazo, 2005; Low \& Simpson, 2012), the absence of distinct labels may have impaired 5-year-olds' ability to move flexibly between their own perspective and that of another person.

Future work could directly investigate the impact of the factors discussed earlier in several ways. First, the similarity of the protagonist's belief to the actual state of affairs in the world and/or the participants' beliefs could be manipulated in the classic changeof-location task by varying the physical similarity (or distance) between hiding locations. Second, future studies could focus on children's performance when the continuous space is subdivided, either perceptually, using visual cues, or conceptually, using linguistic labels.

Prior work has demonstrated that perspective taking in adults is often contaminated by adults' own knowledge. Advancing beyond this prior work, we developed a new task appropriate for both adults and preschoolers that yielded quantitative information (measured in centimeters) regarding the degree to which prior knowledge biases predictions about where another person holding a false belief will search. Although older children (and adults) may perform well on false belief tasks when the hiding places are well marked, delays are minimal, and the choices are dichotomous and highly differentiable, the fact that they are significantly biased by their own privileged first-person information is detectable in our continuous task. Which aspects of our continuous task versus the classic two-choice task contribute to this bias, and what it means for the representational processes involved in the weighing of information when rendering a judgment about another's contradictory view, is of considerable interest for computational models. Bayesian methods have been used to propose a formal model of this internal reasoning process (Goodman et al., 2006). Quantitative methods that can be used across the life span, such as the one presented here will be of use in testing such computational models (Bernstein et al., 2011).

Finally, the fact that our task has features that approximate perspective taking "in the wild" through the use of temporal delay and uncertain hiding locations can be used across a wide range of ages, and provides a quantitative estimate of perspective-taking bias, suggests that it may provide an ecologically valid tool for assessing the nature and scope of developmental changes and individual differences in perspective-taking abilities across the life span, in a range of different populations.

\section{References}

Apperly, I. A., Back, E., Samson, D., \& Grant, L. (2007). The cost of thinking about false beliefs: Evidence from adult performance on a non-inferential theory of mind task. Cognition, 106, 1093-1108.

Apperly, I. A., Warren, F., Andrews, B. J., Grant, J., \& Todd, S. (2011). Developmental continuity in theory of mind: Speed and accuracy of belief-desire reasoning in children and adults. Child Development, 82, 1691-1703. 
Back, E., \& Apperly, I. A. (2010). Two sources of evidence on the non-automaticity of true and false belief ascription. Cognition, 115, 54-70.

Bernstein, D. M., Atance, C., Meltzoff, A. N., \& Loftus, G. R. (2007). Hindsight bias and developing theories of mind. Child Development, 78, 1374-1394.

Bernstein, D. M., Erdfelder, E., Meltzoff, A. N., Peria, W., \& Loftus, G. R. (2011). Hindsight bias from 3 to 95 years of age. Journal of Experimental Psychology: Learning, Memory, and Cognition, 37, 378-391.

Birch, S. A. J., \& Bloom, P. (2004). Understanding children's and adults' limitations in mental state reasoning. Trends in Cognitive Sciences, 8, 255-260.

Birch, S. A. J., \& Bloom, P. (2007). The curse of knowledge in reasoning about false beliefs. Psychological Science, $18,382-386$.

Chambers, J. R., Epley, N., Savitsky, K., \& Windschitl, P. D. (2008). Knowing too much: Using private knowledge to predict how one is viewed by others. Psychological Science, 19, 542-548.

Epley, N., Keysar, B., Van Boven, L., \& Gilovich, T. (2004). Perspective taking as egocentric anchoring and adjustment. Journal of Personality and Social Psychology, 87, 327-339.

Epley, N., Morewedge, C. K., \& Keysar, B. (2004). Perspective taking in children and adults: Equivalent egocentrism but differential correction. Journal of Experimental Social Psychology, 40, 760-768.

Goodman, N. D., Baker, C. L., Bonawitz, E. B., Mansinghka, V. K., Gopnik, A., Wellman, H., et al. (2006). Intuitive theories of mind: A rational approach to false belief. Proceedings of the Twenty-Eighth Annual Conference of the Cognitive Science Society, 1382-1387.

Gopnik, A., \& Astington, J. W. (1988). Children's understanding of representational change and its relation to the understanding of false belief and the appearancereality distinction. Child Development, 59, 26-37.

Huttenlocher, J., Newcombe, N., \& Sandberg, E. H. (1994). The coding of spatial location in young children. Cognitive Psychology, 27, 115-147.

Jacques, S., \& Zelazo, P. D. (2005). Language and the development of cognitive flexibility: Implications for theory of mind. In J. W. Astington \& J. A. Baird (Eds.), Why language matters for theory of mind (pp. 144-162). New York: Oxford University Press.

Keysar, B., Lin, S., \& Barr, D. J. (2003). Limits on theory of mind use in adults. Cognition, 89, 25-41.

Low, J., \& Simpson, S. (2012). Effects of labeling on preschoolers' explicit false belief performance: Outcomes of cognitive flexibility or inhibitory control. Child Development, 83, 1072-1084.

Mitchell, P., Robinson, E. J., Isaacs, J. E., \& Nye, R. M. (1996). Contamination in reasoning about false belief: An instance of realist bias in adults but not children. Cognition, 59, 1-21.

Moll, H., \& Meltzoff, A. N. (2011). How does it look? Level 2 perspective-taking at 36 months of age. Child Development, 82, 661-673.
Nickerson, R. S. (1999). How we know-and sometimes misjudge - what others know: Imputing one's own knowledge to others. Psychological Bulletin, 126, 747-753.

Perner, J. (1991). Understanding the representational mind. Cambridge, MA: MIT Press.

Royzman, E. B., Cassidy, K. W., \& Baron, J. (2003). "I know, you know": Epistemic egocentrism in children and adults. Review of General Psychology, 7, 38-65.

San Juan, V., \& Astington, J. W. (2012). Bridging the gap between implicit and explicit understanding: How language development promotes the processing and representation of false belief. British Journal of Developmental Psychology, 30, 105-122.

Wellman, H. M. (1990). The child's theory of mind. Cambridge, MA: MIT Press.

Wellman, H. M., Cross, D., \& Watson, J. (2001). Metaanalysis of theory-of-mind development: The truth about false belief. Child Development, 72, 655-684.

Wimmer, H., \& Perner, J. (1983). Beliefs about beliefs: Representation and constraining function of wrong beliefs in young children's understanding of deception. Cognition, 13, 103-128.

\section{Appendix}

Experiment 1

\begin{tabular}{lcc}
\hline Trial type & Location A $(\mathrm{cm})$ & Location B $(\mathrm{cm})$ \\
\hline Small distance & 30.5 & 15.2 \\
Large distance & 61 & 25.4 \\
Small distance & 91.4 & 127 \\
Large distance & 121.9 & 137.2 \\
\hline
\end{tabular}

Note. In Experiment 1, small and large distance trials alternated, and the hiding location pairs were counterbalanced for the small and large distance trials. Conditions (no false belief vs. false belief) were administered between subjects.

Experiment 2

\begin{tabular}{lcc}
\hline Trial type & Location A $(\mathrm{cm})$ & Location B $(\mathrm{cm})$ \\
\hline Large distance & 61 & 25.4 \\
Large distance & 45.7 & 81.3 \\
Large distance & 106.7 & 71.1 \\
Large distance & 91.4 & 127 \\
Large distance & 50.8 & 86.4 \\
Large distance & 76.2 & 111.8 \\
Large distance & 121.9 & 86.4 \\
Large distance & 66 & 101.6 \\
Large distance & 111.8 & 76.2 \\
\hline
\end{tabular}

Note. In Experiment 2, four trials were false belief trials, one was a switch trial (always Trial 5), and four trials were memory trials; the order of the false belief and memory trials was counterbalanced. Locations were administered in a fixed order, which is illustrated above. 